\title{
Extremal black holes in the Hořava-Lifshitz gravity
}

\author{
Hyung Won Lee, Yong-Wan Kim, and Yun Soo Myung 1 \\ Institute of Basic Science and School of Computer Aided Science \\ Inje University, Gimhae 621-749, Korea
}

\begin{abstract}
$\underline{\text { Abstract }}$
We study the near-horizon geometry of extremal black holes in the $z=3$ Hořava-Lifshitz gravity with a flow parameter $\lambda$. For $\lambda>1 / 2$, near-horizon geometry of extremal black holes are $\mathrm{AdS}_{2} \times S^{2}$ with different radii, depending on the (modified) Hořava-Lifshitz gravity. For $1 / 3 \leq \lambda \leq 1 / 2$, the radius $v_{2}$ of $S^{2}$ is negative, which means that the near-horizon geometry is ill-defined and the corresponding Bekenstein-Hawking entropy is zero. We show explicitly that the entropy function approach does not work for obtaining the Bekenstein-Hawking entropy of extremal black holes.
\end{abstract}

${ }^{1}$ e-mail address: ysmyung@inje.ac.kr 


\section{Introduction}

Recently Hořava has proposed a renormalizable theory of gravity at a Lifshitz point [1, which may be regarded as a UV complete candidate for general relativity. At short distances the theory of Hořava-Lifshitz (HL) gravity describes interacting nonrelativistic gravitons and is supposed to be power counting renormalizable in $(1+3)$ dimensions. Recently, the HL gravity theory has been intensively investigated in [2], its cosmological applications in [3, 4], and its black hole solutions in [5, 6, 7, 8, 9, 10, 11, 12, 13, 14, 15, 16, 18, 17, 19, 20, 21, 22, 23, 24, 25, 26, 27, 28, 29].

There are two classes of Hořava-Lifshitz gravity in the literature: with/without the projectability condition where the former (latter) implies that the lapse function depends on time (time and space).

Concerning the spherically symmetric solutions without the projectability condition, Lü-Mei-Pope (LMP) have obtained the black hole solution with a flow parameter $\lambda$ [5] and topological black holes were found in [6]. Its thermodynamics were studied in [9, 11] but there remain unclear issues in defining the ADM mass and entropy because its asymptotes are Lifshitz for $1 / 3 \leq \lambda<3(0<z \leq 4)$. On the other hand, Kehagias and Sfetsos (KS) have found the $\lambda=1$ black hole solution in asymptotically flat spacetimes using the modified HL gravity without the projectability condition [10]. Its thermodynamics was defined in [13] but recently, the entropy was argued to take the Bekenstein-Hawking form [30, 31]. Park has obtained a $\lambda=1$ black hole solution with two parameter $\omega$ and $\Lambda_{W}$ [18]. Within the projectable theories, their black hole solutions are less interesting [32].

Before proceeding, we wish to point out that the Hořava black holes (LMP and KS black holes) are completely different from the IR black holes (Schwarzschild-AdS and Schwarzschild black holes). The Hořava black holes have their extremal black holes, whereas the IR black holes do not have extremal black holes. In the Hořava black holes, the chargelike quantities (pseudo charge) are related to the cosmological constant $-\frac{1}{\Lambda_{W}}$ in the LMP black holes and parameter $\frac{1}{2 \omega}$ in the KS black hole. This feature is very special, in compared to the Reissner-Norström-AdS and Reissner-Norström black holes with the electric (magnetic) charge " $Q$ " which were obtained from the relativistic theories. We wish to mention that in the non-relativistic theories, the notion of horizon, temperature, and entropy was not well-defined [33]. Although most solutions have horizons, a part of solutions appears to be horizonless for particles with ultra-luminal dispersion relations.

In order that the thermodynamics of an extremal black hole is explored completely, the whole spacetimes should be known, including the near-horizon geometry and asymptotic 
structures. However, for $1 / 3 \leq \lambda<3$, the LMP black holes have asymptotically Lifshitz which is not yet understood fully. In this sense, we call these Lifshitz black holes. Thus, it is still lack for understanding thermodynamics of the Lifshitz black holes because the conserved quantities are not defined unambiguously.

In this work, we investigate the near-horizon geometry $\left(\mathrm{AdS}_{2} \times S^{2}\right)$ of the extremal black holes to explore the unknown solution in asymptotically flat spacetimes and to understand the Lifshitz black holes. For this purpose, we obtain $\mathrm{AdS}_{2} \times S^{2}$ by exploring the KS solution and the LMP solution. Then, we compare these with curvature radius $v_{1}$ of $\mathrm{AdS}_{2}$ and curvature radius $v_{2}$ of $S^{2}$ obtained by solving full Einstein equations on the $\operatorname{AdS}_{2} \times S^{2}$ background directly. For $\lambda>1 / 2$ case, the near-horizon geometry of extremal black holes are $\mathrm{AdS}_{2} \times S^{2}$ with different curvatures, depending on the (modified) Hořava-Lifshitz gravity. For $1 / 3 \leq \lambda<1 / 2$ case, the radius $v_{2}$ of $S^{2}$ is always negative and thus the corresponding near-horizon geometry is ill-defined. Hence, this case will be ruled out as candidate for the extremal black holes in the Hořava-Lifshitz gravity.

\section{HL gravity}

Introducing the ADM formalism where the metric is parameterized [34]

$$
d s_{A D M}^{2}=-N^{2} d t^{2}+g_{i j}\left(d x^{i}-N^{i} d t\right)\left(d x^{j}-N^{j} d t\right)
$$

the Einstein-Hilbert action can be expressed as

$$
S_{E H}=\frac{1}{16 \pi G} \int d^{4} x \sqrt{g} N\left[K_{i j} K^{i j}-K^{2}+R-2 \Lambda\right]
$$

where $G$ is Newton's constant and extrinsic curvature $K_{i j}$ takes the form

$$
K_{i j}=\frac{1}{2 N}\left(\dot{g}_{i j}-\nabla_{i} N_{j}-\nabla_{j} N_{i}\right) .
$$

Here, a dot denotes a derivative with respect to $t$. An action of the non-relativistic renormalizable gravitational theory is given by [10]

$$
S_{H L}=\int d t d^{3} \mathbf{x}\left[\mathcal{L}_{K}+\mathcal{L}_{V}\right]
$$

where the kinetic term is given by

$$
\mathcal{L}_{K}=\frac{2}{\kappa^{2}} \sqrt{g} N K_{i j} \mathcal{G}^{i j k l} K_{k l}=\frac{2}{\kappa^{2}} \sqrt{g} N\left(K_{i j} K^{i j}-\lambda K^{2}\right),
$$

with the DeWitt metric

$$
\mathcal{G}^{i j k l}=\frac{1}{2}\left(g^{i k} g^{j l}-g^{i l} g^{j k}\right)-\lambda g^{i j} g^{k l}
$$


and its inverse metric

$$
\mathcal{G}_{i j k l}=\frac{1}{2}\left(g_{i k} g_{j l}-g_{i l} g_{j k}\right)-\frac{\lambda}{3 \lambda-1} g_{i j} g_{k l} .
$$

The potential term is determined by the detailed balance condition (DBC) as

$$
\begin{aligned}
\mathcal{L}_{V}=-\frac{\kappa^{2}}{2} \sqrt{g} N E^{i j} \mathcal{G}_{i j k l} E^{k l} & =\sqrt{g} N\left\{\frac{\kappa^{2} \mu^{2}}{8(1-3 \lambda)}\left(\frac{1-4 \lambda}{4} R^{2}+\Lambda_{W} R-3 \Lambda_{W}^{2}\right)\right. \\
& \left.-\frac{\kappa^{2}}{2 \eta^{4}}\left(C_{i j}-\frac{\mu \eta^{2}}{2} R_{i j}\right)\left(C^{i j}-\frac{\mu \eta^{2}}{2} R^{i j}\right)\right\} .
\end{aligned}
$$

Here the $E$ tensor is defined by

$$
E^{i j}=\frac{1}{\eta^{2}} C^{i j}-\frac{\mu}{2}\left(R^{i j}-\frac{R}{2} g^{i j}+\Lambda_{W} g^{i j}\right)
$$

with the Cotton tensor $C_{i j}$

$$
C^{i j}=\frac{\epsilon^{i k \ell}}{\sqrt{g}} \nabla_{k}\left(R_{\ell}^{j}-\frac{1}{4} R \delta_{\ell}^{j}\right) .
$$

Explicitly, $E_{i j}$ could be derived from the Euclidean topologically massive gravity

$$
E^{i j}=\frac{1}{\sqrt{g}} \frac{\delta W_{T M G}}{\delta g_{i j}}
$$

with

$$
W_{T M G}=\frac{1}{\eta^{2}} \int d^{3} x \epsilon^{i j k}\left(\Gamma_{i l}^{m} \partial_{j} \Gamma_{k m}^{l}+\frac{2}{3} \Gamma_{i l}^{n} \Gamma_{j m}^{l} \Gamma_{k n}^{m}\right)-\mu \int d^{3} x \sqrt{g}\left(R-2 \Lambda_{W}\right),
$$

where $\epsilon^{i k l}$ is a tensor density with $\epsilon^{123}=1$. In the IR limit, comparing Eq.(41) with Eq.(2) of general relativity, the speed of light, Newton's constant and the cosmological constant are given by

$$
c=\frac{\kappa^{2} \mu}{4} \sqrt{\frac{\Lambda_{W}}{1-3 \lambda}}, \quad G=\frac{\kappa^{2}}{32 \pi c}, \quad \Lambda=\frac{3}{2} \Lambda_{W} .
$$

The equations of motion were derived in [3] and [5]. We would like to mention that the IR vacuum of this theory is anti de Sitter $\left(\mathrm{AdS}_{4}\right)$ spacetimes. Hence, it is interesting to take a limit of the theory, which may lead to a Minkowski vacuum in the IR sector. To this end, one may deform the theory by introducing a modified term of " $\mu$ " $R$ " $\left(\tilde{\mathcal{L}}_{V}=\mathcal{L}_{V}+\sqrt{g} N \mu^{4} R\right)$ and then, take the $\Lambda_{W} \rightarrow 0$ limit. This does not alter the UV properties of the theory, while it changes the IR properties. That is, there exists a Minkowski vacuum, instead of an AdS vacuum. In the IR limit, the speed of light and Newton's constant are given by

$$
c=\frac{\kappa^{2} \mu^{4}}{2}, G=\frac{\kappa^{2}}{32 \pi c}, \lambda=1 .
$$


Taking $N^{i}=0$ and $K_{i j}=0$, a spherically symmetric solution could be obtained with the metric ansatz

$$
d s_{S S}^{2}=-N^{2}(\rho) d \tau^{2}+\frac{d \rho^{2}}{f(\rho)}+\rho^{2}\left(d \theta^{2}+\sin ^{2} \theta d \phi^{2}\right) .
$$

Substituting the metric ansatz (15) into $\tilde{\mathcal{L}}_{V}$ with $R=-\frac{2}{\rho^{2}}\left(\rho f^{\prime}+f-1\right)$, one has the reduced Lagrangian

$$
\begin{aligned}
\tilde{\mathcal{L}}_{V}^{S S} & =\frac{\kappa^{2} \mu^{2} N}{8(1-3 \lambda) \sqrt{f}}\left[\frac{\lambda-1}{2} f^{\prime 2}-\frac{2 \lambda(f-1)}{\rho} f^{\prime}\right. \\
& \left.+\frac{(2 \lambda-1)(f-1)^{2}}{\rho^{2}}-2\left(w-\Lambda_{W}\right)\left(1-f-\rho f^{\prime}\right)-3 \Lambda_{W}^{2} \rho^{2}\right] .
\end{aligned}
$$

Here the parameter $\omega$ controls the UV effects

$$
w=\frac{8 \mu^{2}(3 \lambda-1)}{\kappa^{2}}
$$

which is positive for $\lambda>1 / 3$. This is possible because the metric ansatz shows all the allowed singlets which are compatible with the $S O(3)$ action on the $S^{2}$. In other words, the ADM decomposition of HL gravity implies naturally the presence of a spherically symmetric static solution, in addition to the time-evolving solution of hypersurfaces for foliation preserving diffeomorphisms [1].

\section{Kehagias-Sfetsos black hole}

For $\lambda=1\left(w=16 \mu^{2} / \kappa^{2} \equiv 1 / 2 Q^{2}\right)$ and $\Lambda_{W}=0$, we have Kehagias-Sfetsos (KS) black hole solution where $f$ and $N$ are determined to be

$$
N^{2}=f=\frac{2\left(\rho^{2}-2 M \rho+Q^{2}\right)}{\rho^{2}+2 Q^{2}+\sqrt{\rho^{4}+8 Q^{2} M \rho}} .
$$

Here $M$ may be related to the ADM mass. It seems that the metric function $f$ looks like that of Reissner-Nordström (RN) black hole. The outer (event) and inner (Cauchy) horizons are given by

$$
\rho_{ \pm}=M \pm \sqrt{M^{2}-Q^{2}}
$$

which is the same form as in the RN black hole. The extremal black hole is located at

$$
\rho_{ \pm}=\rho_{e}=M=Q
$$

This is why we take $\frac{1}{2 \omega}$ to be the charge-like quantity (pseudo charge) $Q^{2}$. In the limit of $Q^{2} \rightarrow 0(\omega \rightarrow \infty)$, we recover the Schwarzschild black hole as the IR black hole. 
However, for large $\rho$, we approximate the metric function as

$$
f \rightarrow 1-\frac{2 M}{\rho}+\frac{4 M^{2} Q^{2}}{\rho^{4}}+\cdots,
$$

which is different from the RN-metric function

$$
f_{R N}=1-\frac{2 M}{\rho}+\frac{Q^{2}}{\rho^{2}} .
$$

At this stage, we define the Bekenstein-Hawking entropy for the extremal KS black hole2 as

$$
S_{\mathrm{BH}}=\frac{A}{4}=\pi Q^{2}
$$

In order to explore the near-horizon geometry $A d S_{2} \times S^{2}$ of extremal black hole, we introduce new coordinates $t$ and $r$ using relations

$$
t=\frac{\epsilon}{3 Q^{2}} \tau, \quad r=\frac{\rho-Q}{\epsilon},
$$

where $\epsilon$ is an arbitrary constant. Then, the extremal solution can be expressed by new coordinates as

$$
\begin{aligned}
d s_{\mathrm{ext}}^{2}= & -\frac{2 r^{2}}{(Q+\epsilon r)^{2}+2 Q^{2}+\sqrt{(Q+\epsilon r)^{4}+8 Q^{3}(Q+\epsilon r)}} 9 Q^{4} d t^{2} \\
& +\frac{(Q+\epsilon r)^{2}+2 Q^{2}+\sqrt{(Q+\epsilon r)^{4}+8 Q^{3}(Q+\epsilon r)}}{2 r^{2}} d r^{2}+(Q+\epsilon r)^{2} d \Omega_{2}^{2} .
\end{aligned}
$$

Taking the 'near-horizon' limit of $\epsilon \rightarrow 0$ leads to

$$
d s_{\mathrm{KS}}^{2}=v_{1}\left(-r^{2} d t^{2}+\frac{d r^{2}}{r^{2}}\right)+v_{2} d \Omega_{2}^{2}
$$

with

$$
v_{1}=3 Q^{2}, \quad v_{2}=Q^{2}
$$

This shows clearly $A d S_{2} \times S^{2}$ in the Poincare coordinates. Its curvature is given by

$$
{ }^{(4)} R_{\mathrm{KS}}=R_{A d S_{2}}+R_{S^{2}}=-\frac{2}{3 Q^{2}}+\frac{2}{Q^{2}}
$$

\footnotetext{
${ }^{2}$ At present, we may have two kinds of entropy: one is the logarithmic entropy of $S=\frac{A}{4}+\frac{\pi}{\omega} \ln \left[\frac{A}{4}\right][20,35]$ and the other is the Bekenstein-Hawking entropy $S_{B H}=\frac{A}{4}$ [30, 31]. The former can be obtained from the first law of thermodynamics $d M=T_{H} d S$ provided $M$ and $T_{H}$ are known. The temperature is defined from the surface gravity at the horizon and thus, it is independent of the asymptotic structure. On the other hand, the ADM mass $M$ depends on the asymptotic structure because it belongs to a conserved quantity defined at infinity. Hence, the entropy also depends on the mass $M$. However, it is hard to accept the logarithmic entropy without considering quantum or thermal corrections. Therefore, it would be better to use the area-law of the Bekenstein-Hawking entropy to derive the ADM mass using the first law. In this work, we choose the Bekenstein-Hawking entropy as the entropy of the Hořava black holes.
} 
In this case, the KS black hole solution interpolates between $A d S_{2} \times S^{2}$ in the near-horizon geometry and Minkowski spacetimes at asymptotic infinity.

However, the RN black hole has the Bertotti-Robinson metric as its near-horizon geometry

$$
d s_{\mathrm{RN}}^{2}=Q^{2}\left(-r^{2} d t^{2}+\frac{d r^{2}}{r^{2}}\right)+Q^{2} d \Omega_{2}^{2}
$$

Its curvature is given by

$$
{ }^{(4)} R_{\mathrm{RN}}=-\frac{2}{Q^{2}}+\frac{2}{Q^{2}} .
$$

Finally, we mention that the general solution with $\lambda$ is not found in asymptotically flat spacetimes because third-order derivatives make it difficult to solve the Einstein equation.

\section{Lü-Mei-Pope black hole solution}

In this case, we take $\omega=0$ in Eq. (16) by dropping the modified term of $\mu^{4} R$. The Lü-Mei-Pope (LMP) solutions for $z=3$ Hořava-Lifshitz gravity are given by

$$
f(x)=1+x^{2}-\alpha x^{p_{ \pm}(\lambda)}, \quad N(x)=x^{q_{ \pm}(\lambda)} \sqrt{f(x)},
$$

where

$$
x=\sqrt{-\Lambda_{W}} r, p_{ \pm}(\lambda)=\frac{2 \lambda \pm \sqrt{6 \lambda-2}}{\lambda-1}, \quad q_{ \pm}(\lambda)=-\frac{1+3 \lambda \pm 2 \sqrt{6 \lambda-2}}{\lambda-1} .
$$

In this work, we choose $p_{-}(\lambda)=p(\lambda)$ and $q_{-}(\lambda)=q(\lambda)$ only. Its extremal black hole with $f\left(x_{e}\right)=0$ and $f^{\prime}\left(x_{e}\right)=0$ are located as

$$
x_{e}=0, \text { for } \frac{1}{3} \leq \lambda \leq \frac{1}{2} ; \quad x_{e}=\sqrt{\frac{p(\lambda)}{2-p(\lambda)}}=\sqrt{\frac{2 \lambda-\sqrt{6 \lambda-2}}{-2+\sqrt{6 \lambda-2}}}, \text { for } \lambda>\frac{1}{2} .
$$

The Bekenstein-Hawking entropy for the extremal LMP black holes is defined by

$$
S_{\mathrm{BH}}=\pi x_{e}^{2}\left[\frac{1}{-\Lambda_{W}}\right]
$$

where $-\frac{1}{\Lambda_{W}}$ is interpreted as a pseudo charge. Hence the non-zero entropy is available for $\lambda>1 / 2$ only. The metric function $f(x)$ can be expanded around extremal point to find its near-horizon geometry $\mathrm{AdS}_{2} \times S^{2}$ as

$$
f(x) \approx \frac{f^{\prime \prime}\left(x_{e}\right)}{2}(\epsilon \rho)^{2}+\mathcal{O}\left(\epsilon^{3}\right)
$$

where $x-x_{e}=\epsilon \rho$ and $\tilde{t}=\epsilon A t$ with

$$
A=\frac{f^{\prime \prime}\left(x_{e}\right) x_{e}^{q(\lambda)}}{2} \sqrt{-\Lambda_{W}}
$$


The line element is given by [19]

$$
d s^{2}=\frac{2}{f^{\prime \prime}\left(x_{e}\right)}\left[\frac{1}{-\Lambda_{W}}\right]\left(-\rho^{2} d \tilde{t}^{2}+\frac{d \rho^{2}}{\rho^{2}}\right)+\left[\frac{x_{e}^{2}}{-\Lambda_{W}}\right] d \Omega^{2}
$$

with

$$
f^{\prime \prime}\left(x_{e}\right)=4-2 p(\lambda)
$$

Comparing the above expression with $\mathrm{AdS}_{2} \times S^{2}$, we obtain

$$
\begin{aligned}
& v_{1}=\frac{1}{2-p(\lambda)}\left[\frac{1}{-\Lambda_{W}}\right]=\frac{v_{2}}{p(\lambda)}, \\
& v_{2}=\frac{p(\lambda)}{2-p(\lambda)}\left[\frac{1}{-\Lambda_{W}}\right] .
\end{aligned}
$$

We express the Bekenstein-Hawking entropy as $S_{\mathrm{BH}}=\pi v_{2}$. For $\lambda=1$, we obtain the LMP case

$$
x_{e}^{2}=\frac{1}{3}, \quad v_{1}=\frac{2}{3}\left[\frac{1}{-\Lambda_{W}}\right]=2 v_{2}, \quad v_{2}=\frac{1}{3}\left[\frac{1}{-\Lambda_{W}}\right] .
$$

Importantly, from Fig. 1, it is problematic to define the near-horizon geometry of extremal black hole for $1 / 3 \leq \lambda \leq 1 / 2$ because $v_{2}$ is negative for $1 / 3 \leq \lambda<1 / 2(p(\lambda)<0)$ and $v_{2}$ is zero for $\lambda=1 / 2(p(\lambda)=0)$. That is, its near-horizon geometry is ill-defined. In addition, the Bekenstein-Hawking entropy is zero.

It seems that the Bertotti-Robinson geometry ( $\mathrm{RN}$ black hole) of $v_{1}=v_{2}=-1 / \Lambda_{W}$ is recovered from $\lambda=3$ LMP black hole with $x_{e}^{2}=1$

$$
f(x)=1+x^{2}-\alpha x, \quad N(x)=\frac{\sqrt{f(x)}}{x} .
$$

However, its asymptotic spacetimes are completely different from asymptotically flat spacetimes of the RN black hole.

For $1 / 2<\lambda<3$, the LMP black hole solutions interpolate between $\mathrm{AdS}_{2} \times S^{2}$ with $v_{1}>v_{2}$ in the near-horizon geometry of extremal black hole and Lifshitz at asymptotic infinity, while for $\lambda>3$, these interpolate $\mathrm{AdS}_{2} \times S^{2}$ with $v_{1}<v_{2}$ in the near-horizon and Minkowski spacetimes at asymptotic infinity.

\section{Solution to full equations on $\mathbf{A d S}_{2} \times S^{2}$}

In this section, we wish to find $v_{1}$-and $v_{2}$-solutions by solving full Einstein equations on the $\mathrm{AdS}_{2} \times S^{2}$ background with $\mathcal{L}_{K}=0$. This study will be very useful for a further work on Hořava black holes because it may provide a hint to explore the unknown black hole solutions. 


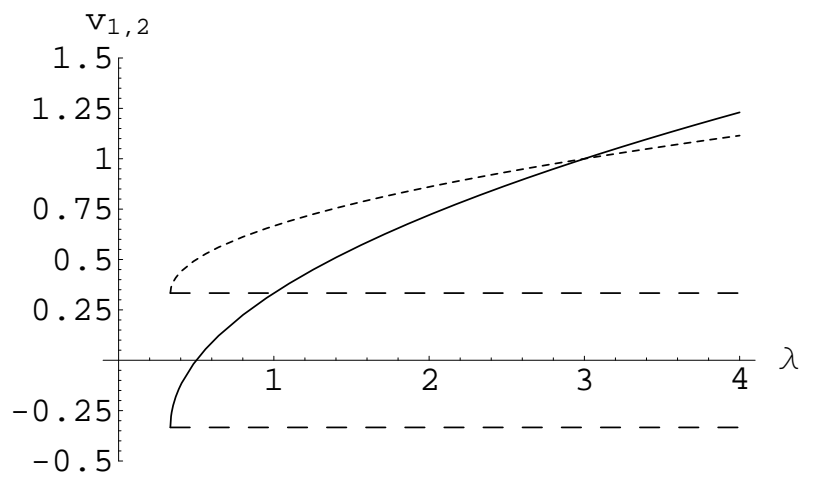

Figure 1: $v_{1}(\lambda)$ (dotted curves) and $v_{2}(\lambda)$ (solid curves) graphs with $\Lambda_{W}=-1$. We find $v_{1}(1 / 3)=1 / 3, v_{1}(1 / 2)=1 / 2, v_{1}(1)=2 / 3$, and $v_{1}(3)=1$, while we observe $v_{2}(1 / 3)=$ $-1 / 3, v_{2}(1 / 2)=0, v_{2}(1)=1 / 3$, and $v_{2}(3)=1$.

A variation to $\tilde{\mathcal{L}}_{V}$ with respect to $N$ is modified as

$$
\begin{aligned}
\frac{\delta \tilde{\mathcal{L}}_{V}}{\delta N}=0: \sqrt{g}\left[\frac{\kappa^{2} \mu^{2}\left(\Lambda_{W}-\omega\right)}{8(1-3 \lambda)} R\right. & -\frac{3 \kappa^{2} \mu^{2} \Lambda_{W}^{2}}{8(1-3 \lambda)} \\
& \left.+\frac{\kappa^{2} \mu^{2}(1-4 \lambda)}{32(1-3 \lambda)} R^{2}-\frac{\kappa^{2}}{2 \eta^{4}}\left(C_{i j}-\frac{\mu \eta^{2}}{2} R_{i j}\right)^{2}\right]=0 .
\end{aligned}
$$

A variation to with respect to the shift function $N^{j}$ is trivial for finding a black hole solution as

$$
\frac{\delta \tilde{\mathcal{L}}_{V}}{\delta N^{i}}=0 \rightarrow \text { trivial }
$$

A variation to $\mathcal{L}_{K}+\tilde{\mathcal{L}}_{V}$ with respect to $g_{i j}$ is changed to

$$
\frac{\delta \tilde{\mathcal{L}}_{V}}{\delta g^{i j}}=0: \mathcal{E}_{i j} \equiv \frac{\kappa^{2} \mu^{2}\left(\Lambda_{W}-\omega\right)}{8(1-3 \lambda)} E_{i j}^{(3)}+\frac{\kappa^{2} \mu^{2}(1-4 \lambda)}{32(1-3 \lambda)} E_{i j}^{(4)}-\frac{\kappa^{2} \mu}{4 \eta^{2}} E_{i j}^{(5)}-\frac{\kappa^{2}}{2 \eta^{4}} E_{i j}^{(6)}=0
$$

where $E_{i j}^{(3)}$ takes the modified form

$$
E_{i j}^{(3)}=N\left(R_{i j}-\frac{1}{2} R g_{i j}+\frac{3}{2} \frac{\Lambda_{W}^{2}}{\Lambda_{W}-\omega} g_{i j}\right)-\left(\nabla_{i} \nabla_{j}-g_{i j} \nabla^{2}\right) N
$$

and all remaining terms are the same as in [5].

Considering $\mathrm{AdS}_{2} \times S^{2}$ in Eq. (26), we have

$$
\begin{aligned}
N & =\sqrt{v_{1}} r, \quad g_{i j}=\operatorname{diag}\left[\frac{v_{1}}{r^{2}}, v_{2}, v_{2} \sin ^{2} \theta\right] \\
R_{i j} & =\operatorname{diag}\left[0,1, \sin ^{2} \theta\right], C_{i j}=\operatorname{diag}[0,0,0], K_{i j}=\operatorname{diag}[0,0,0] \\
R & =\frac{2}{v_{2}}, \quad K=0 .
\end{aligned}
$$


The $N$-variation leads to the equation obtained when varying with respect to $v_{1}$ as

$$
\frac{\kappa^{2} \mu^{2} \sqrt{v_{1}} \sin \theta}{8(3 \lambda-1) v_{2} r}\left[3 \Lambda_{W}^{2} v_{2}^{2}+2\left(\omega-\Lambda_{W}\right) v_{2}-2 \lambda+1\right]=0 .
$$

The $g_{i j}$-variation leads to three component equations

$$
\begin{aligned}
\mathcal{E}_{r r} & =\frac{\kappa^{2} \mu^{2} v_{1}^{3 / 2}}{16(3 \lambda-1) v_{2}^{2} r}\left[3 \Lambda_{W}^{2} v_{2}^{2}+2\left(\omega-\Lambda_{W}\right) v_{2}-2 \lambda+1\right]=0 \\
\mathcal{E}_{\theta \theta} & =\frac{\kappa^{2} \mu^{2} r}{16(3 \lambda-1) v_{1}^{1 / 2} v_{2}}\left[v_{1}\left(3 \Lambda_{W}^{2} v_{2}^{2}+2 \lambda-1\right)-2\left(\left(\omega-\Lambda_{W}\right) v_{2}+\lambda\right) v_{2}\right]=0 \\
\mathcal{E}_{\phi \phi} & =\sin ^{2} \theta \mathcal{E}_{\theta \theta}=0
\end{aligned}
$$

Eqs. (48) and (49) give the same equation for $v_{2}$ as

$$
3 \Lambda_{W}^{2} v_{2}^{2}+2\left(\omega-\Lambda_{W}\right) v_{2}-2 \lambda+1=0
$$

For $\Lambda_{W} \neq 0$, this equation is solved to give

$$
\Lambda_{W} v_{2}=\frac{1}{3}\left(1-\frac{\omega}{\Lambda_{W}}\right)\left(1 \pm \sqrt{1+\frac{6 \lambda-3}{\left(1-\frac{\omega}{\Lambda_{W}}\right)^{2}}}\right)
$$

For $\Lambda_{W}<0$, the solution is

$$
v_{2}\left(\lambda, \omega, \Lambda_{W}\right)=\frac{1}{3}\left(1-\frac{\omega}{\Lambda_{W}}\right)\left(\sqrt{1+\frac{6 \lambda-3}{\left(1-\frac{\omega}{\Lambda_{W}}\right)^{2}}}-1\right)\left[\frac{1}{-\Lambda_{W}}\right] .
$$

From Eq. (50), we find for $\Lambda_{W} \neq 0$

$$
v_{1}\left(\lambda, \omega, \Lambda_{W}\right)=\frac{6 \lambda-2\left(1-\frac{\omega}{\Lambda_{W}}\right)^{2}\left(1-\sqrt{1+\frac{6 \lambda-3}{\left(1-\frac{\omega}{\Lambda_{W}}\right)^{2}}}\right)}{6 \lambda-3+\left(1-\frac{\omega}{\Lambda_{W}}\right)^{2}\left(1-\sqrt{1+\frac{6 \lambda-3}{\left(1-\frac{\omega}{\Lambda_{W}}\right)^{2}}}\right)^{2}} v_{2}\left(\lambda, \omega, \Lambda_{W}\right) .
$$

Eqs. (54) and (55) are our main results.

\subsection{General KS $\operatorname{AdS}_{2} \times S^{2}$-solution}

For $\Lambda_{W}=0$, Eq. (52) becomes

$$
2 \omega v_{2}-2 \lambda+1=0
$$

which gives the solution

$$
v_{2}=\frac{2 \lambda-1}{2 \omega}
$$


From Eq. (50) with Eq. (57), one has the relation between $v_{1}$ and $v_{2}$

$$
v_{1}=\frac{4 \lambda-1}{2 \lambda-1} v_{2}
$$

The Bekenstein-Hawking entropy for extremal black holes with $\lambda$ may take

$$
S_{\mathrm{BH}}=\pi v_{2}=\pi\left[\frac{2 \lambda-1}{2 \omega}\right] .
$$

For $\lambda=1$, we recover the $\mathrm{KS} \mathrm{AdS}_{2} \times S^{2}$ exactly as

$$
v_{1}=3 v_{2}, \quad v_{2}=\frac{1}{2 \omega}=Q^{2}
$$

whose entropy leads to Eq. (23). Although the black hole solution with arbitrary $\lambda$ is not yet known in asymptotically flat spacetimes, its near-horizon geometry of extremal black hole with $\lambda$ could be found from Eqs. (57) and (58). For $\lambda>1 / 2$, we can define extremal black holes because both $v_{1}$ and $v_{2}$ are positive.

\section{2 $\quad$ LMP $\mathrm{AdS}_{2} \times S^{2}$ solution}

Here we comment on the $\omega=0$ case. Eq. (54) provides the solution $v_{2}$

$$
v_{2}=\frac{\sqrt{6 \lambda-2}-1}{3}\left[\frac{1}{-\Lambda_{W}}\right]=\left[\frac{p(\lambda)}{2-p(\lambda)}\right]\left[\frac{1}{-\Lambda_{W}}\right] .
$$

From Eq. (55) with $\omega=0$, we obtain

$$
v_{1}=\frac{\lambda-1}{2 \lambda-\sqrt{6 \lambda-2}} v_{2}=\frac{v_{2}}{p(\lambda)} .
$$

This is the same result as was found from the previous section.

\subsection{General $\mathrm{AdS}_{2} \times S^{2}$ solution}

We consider the general case of $\omega \neq 0$ and $\Lambda_{W} \neq 0$. As is shown in Fig. 2, it is observed that $v_{2}>0$ only for $\lambda>1 / 2$, irrespective of any values of $\omega$ and $\Lambda_{W}<0$. It could be confirmed from the fact that $v_{2}=0$ implies $\lambda=1 / 2$. We note that the $\lambda=1$ solution describes the near-horizon geometry of Park's solution [18. Also, from Fig. 3, we confirm that $v_{1}$ is always positive.

Finally, the entropy of extremal black hole is determined to be

$$
S_{\mathrm{BH}}=\pi v_{2}
$$

where $v_{2}$ is given by Eq. (54). 


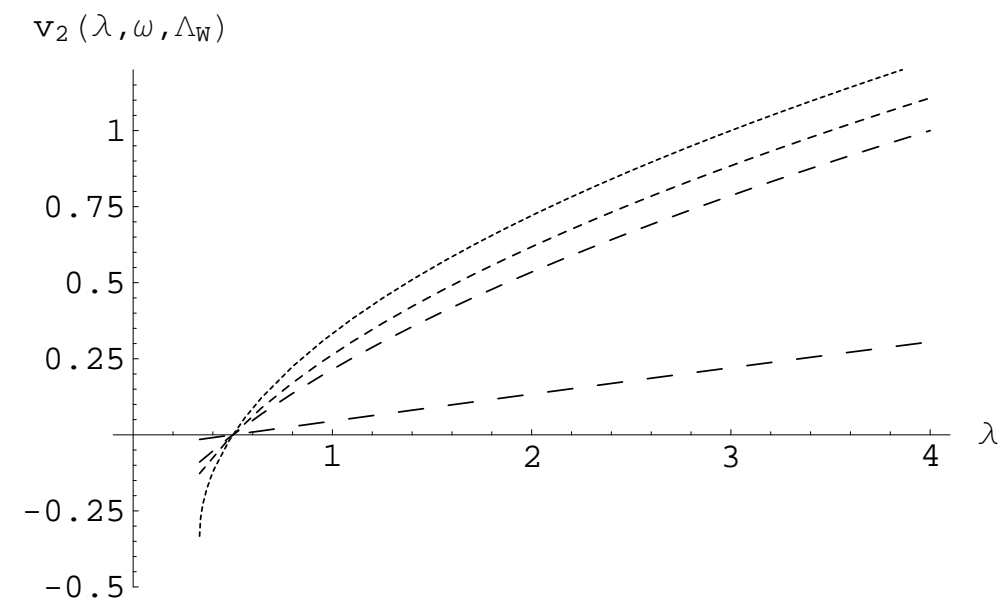

Figure 2: $v_{2}\left(\lambda, \omega, \Lambda_{W}\right)$ graphs with $\Lambda_{W}=-1$. We find that $v_{2}>0,=0,<0$ for $\lambda>\frac{1}{2},=$ $\frac{1}{2},<\frac{1}{2}$, respectively. Four graphs show for $\omega=0,0.5,1$, and 10 from top to bottom.

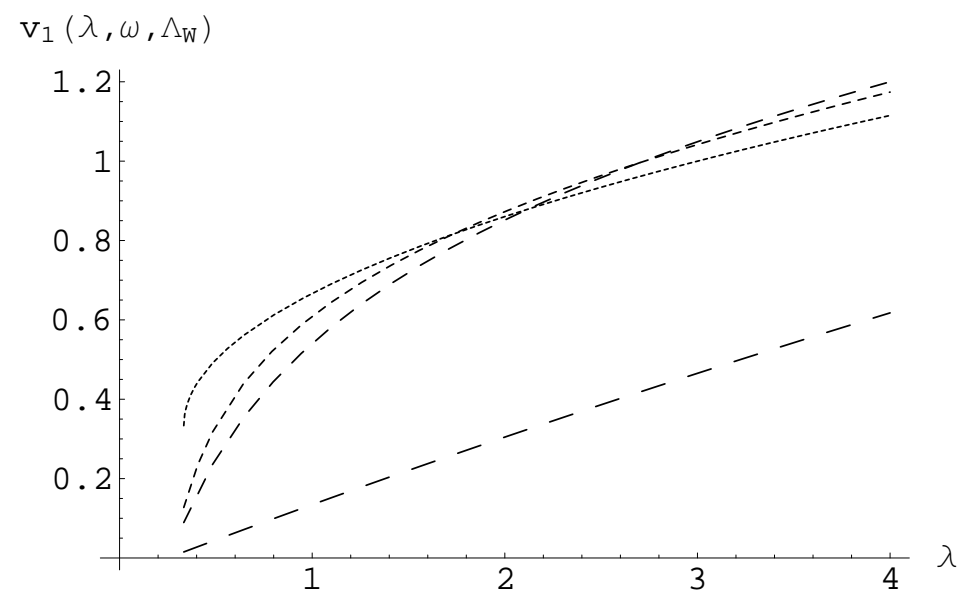

Figure 3: $v_{1}\left(\lambda, \omega, \Lambda_{W}\right)$ graphs with $\Lambda_{W}=-1$. It shows that $v_{1}>0$ for $\lambda>\frac{1}{3}$. Four graphs are for $\omega=0,0.5,1$, and 10 from top to bottom (along $v_{1}$-axis).

\section{Entropy function approach}

Plugging Eq. (47) into $\tilde{\mathcal{L}}_{V}$ leads to the Lagrangian on $\mathrm{AdS}_{2} \times S^{2}$

$$
\tilde{\mathcal{L}}_{V}^{A d S_{2} \times S^{2}}=\frac{\kappa^{2} \mu^{2} v_{1} \sin \theta}{8(3 \lambda-1) v_{2}}\left(3 \Lambda_{W}^{2} v_{2}^{2}+2\left(\omega-\Lambda_{W}\right) v_{2}-2 \lambda+1\right) .
$$

which is nothing but

$$
N \frac{\delta \tilde{\mathcal{L}}_{V}}{\delta N}
$$


in Eq.(48). After integration over $S^{2}$, it takes the form of entropy function 36

$$
\tilde{\mathcal{L}}_{V}^{A d S_{2}}=\frac{\pi \kappa^{2} \mu^{2} v_{1}}{2(3 \lambda-1) v_{2}}\left[3 \Lambda_{W}^{2} v_{2}^{2}+2\left(\omega-\Lambda_{W}\right) v_{2}-2 \lambda+1\right] .
$$

A variation of $\tilde{\mathcal{L}}_{V}^{A d S_{2}}$ with respect to $v_{1}$ leads to the known equation (52)

$$
\frac{\pi \kappa^{2} \mu^{2}}{2(3 \lambda-1) v_{2}}\left[3 \Lambda_{W}^{2} v_{2}^{2}+2\left(\omega-\Lambda_{W}\right) v_{2}-2 \lambda+1\right]=0
$$

while a variation with respect to $v_{2}$ takes a different form

$$
3 \Lambda_{W}^{2} v_{2}^{2}+(2 \lambda-1)=0
$$

The entropy function $\tilde{\mathcal{L}}_{V}^{A d S_{2}}$ is zero if Eq. (67) is used. Since this function contains $v_{1}$ as a global factor, we cannot determine $v_{1}$ by varying the entropy function with respect to $v_{2}$. It seems that this is a handicap in the entropy function approach to the Horaava black holes because the HL gravity is a non-relativistic theory. However, we have determined $v_{1}$ in Eq. (54) when using full Einstein equations on the $\mathrm{AdS}_{2} \times S^{2}$ background. Now we wish to clarify why the reduced Lagrangian (66) is not useful to determine $v_{1}$. The entropy function approach is equivalent to taking into account (48) and (49). On the other hand, the full Einstein equation on the $\mathrm{AdS}_{2} \times S^{2}$ provides two more equations (50) and (151) than the entropy function approach. These determine a relation between $v_{2}$ and $v_{1}$, as is shown in Eq.(55).

\section{Discussions}

In this work, we have studied near-horizon geometry $\mathrm{AdS}_{2} \times S^{2}$ of HL black holes. An important relation is $v_{1}=\frac{v_{2}}{p}$ and $v_{2}=\frac{p}{2-p}\left[\frac{1}{-\Lambda_{W}}\right]$ for the LMP black holes [5]. Also we have obtained $v_{1}=\frac{4 \lambda-1}{2 \lambda-1} v_{2}$ and $v_{2}=\frac{2 \lambda-1}{2 \omega}$ for generalized KS black holes. The $\lambda=1$ case

corresponds to the KS solution: $v_{1}=3 v_{2}, v_{2}=\frac{1}{2 \omega}$ [10]. We regard $\left(-\frac{1}{\Lambda_{W}}, \frac{1}{2 \omega}\right)$ as pseudo charges as magnetic charge " $Q$ " in RN and RN-AdS black holes.

Since $v_{2}$ is zero for $\lambda=1 / 2$, we have to classify whole extremal black holes according to the $\lambda$-value. For $\lambda>1 / 2$ case, near-horizon geometry of extremal black holes are $\mathrm{AdS}_{2} \times S^{2}$ with different curvatures, depending on the (modified) Hořava-Lifshitz gravity. For $1 / 3 \leq$ $\lambda<1 / 2$ case, a radius $v_{2}$ of $S^{2}$ is negative and thus the corresponding near-horizon geometry is ill-defined. Hence this case should be discriminated from the extremal black holes in the Hořava-Lifshitz gravity. This is clear because as is shown in Eq.(33), the Lifshitz black holes with $1 / 3 \leq \lambda \leq 1 / 2(4 \leq z \leq 2)$ have degenerate horizons located at the origin like massless BTZ black hole in three dimensions. 
We hope that the black hole with arbitrary $\lambda$ could be found soon in asymptotically flat spacetimes because its near-horizon geometry of extremal black hole are known as Eqs. (57) and (58). Also, we expect to obtain a general black hole whose near-horizon geometry is given by Eqs. (54) and (55) soon.

Finally, the entropy function approach does not work for obtaining the BekensteinHawking entropy of extremal Hořava black holes because the Lorentz-symmetry was broken.

Noted added-After the appearance of this work in arxiv, considerable research has indicated that for $1 / 3 \leq \lambda \leq 1$, the projectable version of HL gravity has a serious problem called as strong coupled problem. In the projectable theories, the authors [37, 38] have first argued that $\psi$ is propagating around the Minkowski space but it has a negative kinetic term, showing a ghost instability. In this case, the Hořava scalar becomes ghost if the sound speed square $\left(c_{\psi}^{2}\right)$ is positive. In order to make this scalar healthy, the sound speed square must be negative, but it is inevitably unstable. Thus, one way to avoid this is to choose the case that the sound speed square is close to zero, which implies the limit of $\lambda \rightarrow 1$. However, in this limit, the cubic interactions are important at very low energies which indicates the strong coupled problem [39]. This invalidates any linearized analysis and any predictability of quantum gravity is lost due to unsuppressed loop corrections. The authors [40] tried to extend the theory to make a healthy HL gravity, but there has been some debate as to whether this theory is really healthy [41, 42, 43].

On the other hand, in the Hamiltonian approach to the nonprojectable HL gravity, the authors [44] did not consider the Hamiltonian constraint as a second class constraint, which leads to a strange result that there are no degrees of freedom left when imposing the constraints of the theory. Moreover, the authors [45] have claimed that there are no solution of the lapse function which satisfies the constraints. Unfortunately, it implies a surprising conclusion that there is no evolution at all for any observable. However, more recently, it was shown that the IR version of HL gravity ( $\lambda R$-model) is completely equivalent to the general relativity for any $\lambda$ when employing a consistent Hamiltonian formalism based on Dirac algorithm [46].

The projectability condition from condensed matter physics may not be appropriate for describing the (quantum) gravity. Instead, if one does not impose the projectability condition, the HL gravity leads to general relativity without the strong coupling problem in the IR limit. We note that this work was carried out without the projectability condition and thus, was nothing to do with the strong coupling problem for $1 / 3 \leq \lambda \leq 1$. 


\section{Acknowledgement}

Y. Myung thanks Li-Ming Cao, Sinji Mukohyama, and Mu-In Park for helpful discussions.

H. Lee was supported by the National Research Foundation of Korea (NRF) grant funded by the Korea government (MEST) (No. 2009-0062869). Y. Kim was supported by the Korea Research Foundation Grant funded by Korea Government (MOEHRD): KRF-2007359-C00007. Y. Myung was supported by Basic Science Research Program through the National Research Foundation (NRF) of Korea funded by the Ministry of Education, Science and Technology (2009-0086861).

\section{References}

[1] P. Horava, Phys. Rev. D 79, 084008 (2009) [arXiv:0901.3775 [hep-th]].

[2] P. Horava, JHEP 0903, 020 (2009) [arXiv:0812.4287 [hep-th]]; M. Visser, Phys. Rev. D 80, 025011 (2009) arXiv:0902.0590 [hep-th]]; P. Horava, Phys. Rev. Lett. 102, 161301 (2009) arXiv:0902.3657 [hep-th]]; A. Volovich and C. Wen, JHEP 0905, 087 (2009) arXiv:0903.2455 [hep-th]]; J. Kluson, JHEP 0907, 079 (2009) arXiv:0904.1343 [hep-th]]; H. Nikolic, arXiv:0904.3412 [hep-th]; H. Nastase, arXiv:0904.3604 [hep-th]; K. I. Izawa, arXiv:0904.3593 [hep-th]; G. E. Volovik, JETP Lett. 89, 525 (2009) arXiv:0904.4113 [gr-qc]]; T. P. Sotiriou, M. Visser and S. Weinfurtner, Phys. Rev. Lett. 102, 251601 (2009) arXiv:0904.4464 [hep-th]]; B. Chen and Q. G. Huang, Phys. Lett. B 683, 108 (2010) arXiv:0904.4565 [hep-th]]; R. G. Cai, B. Hu and H. B. Zhang, Phys. Rev. D 80, 041501 (2009) arXiv:0905.0255 [hep-th]]; T. Nishioka, Class. Quant. Grav. 26, 242001 (2009) arXiv:0905.0473 [hep-th]]; D. Orlando and S. Reffert, Class. Quant. Grav. 26, 155021 (2009) arXiv:0905.0301 [hep-th]]; C. Charmousis, G. Niz, A. Padilla and P. M. Saffin, JHEP 0908 (2009) 070 arXiv:0905.2579 [hep-th]]; Y. W. Kim, H. W. Lee and Y. S. Myung, Phys. Lett. B 682, 246 (2009) [arXiv:0905.3423 [hep-th]]; R. A. Konoplya, Phys. Lett. B 679, 499 (2009) arXiv:0905.1523 [hep-th]]; G. Calcagni, Phys. Rev. D 81 (2010) 044006 [arXiv:0905.3740 [hep-th]]; M. Sakamoto, Phys. Rev. D 79, 124038 (2009) arXiv:0905.4326 [hep-th]]; Y. S. Myung, Phys. Rev. D 81 (2010) 064006 [arXiv:0906.0848 [hep-th]]; C. Bogdanos and E. N. Saridakis, Class. Quant. Grav. 27 (2010) 075005 arXiv:0907.1636 [hep-th]].

[3] E. Kiritsis and G. Kofinas, Nucl. Phys. B 821, 467 (2009) arXiv:0904.1334 [hep-th]]. 
[4] G. Calcagni, JHEP 0909, 112 (2009) arXiv:0904.0829 [hep-th]]; T. Takahashi and J. Soda, Phys. Rev. Lett. 102, 231301 (2009) [arXiv:0904.0554 [hep-th]]; S. Mukohyama, JCAP 0906, 001 (2009) [arXiv:0904.2190 [hep-th]]; R. Brandenberger, Phys. Rev. D 80 (2009) 043516 arXiv:0904.2835 [hep-th]]; Y. S. Piao, Phys. Lett. B 681 (2009) 1 arXiv:0904.4117 [hep-th]]; X. Gao, arXiv:0904.4187 [hep-th]; B. Chen, S. Pi and J. Z. Tang, JCAP 0908, 007 (2009) arXiv:0905.2300 [hepth]]; E. N. Saridakis, arXiv:0905.3532 [hep-th]; S. Mukohyama, Phys. Rev. D 80, 064005 (2009) arXiv:0905.3563 [hep-th]]; M. Minamitsuji, Phys. Lett. B 684 (2010) 194 arXiv:0905.3892 [astro-ph.CO]]; A. Wang and Y. Wu, JCAP 0907, 012 (2009) arXiv:0905.4117 [hep-th]]; S. Nojiri and S. D. Odintsov, Phys. Rev. D 81 (2010) 043001 arXiv:0905.4213 [hep-th]]; X. Gao, Y. Wang, R. Brandenberger and A. Riotto, Phys. Rev. D 81 (2010) 083508 [arXiv:0905.3821 [hep-th]]; M. I. Park, JCAP 1001, 001 (2010) arXiv:0906.4275 [hep-th]]; S. Mukohyama, JCAP 0909, 005 (2009) arXiv:0906.5069 [hep-th]]; A. Wang and R. Maartens, Phys. Rev. D 81, 024009 (2010) arXiv:0907.1748 [hep-th]]; S. S. Kim, T. Kim and Y. Kim, Phys. Rev. D 80 (2009) 124002 [arXiv:0907.3093 [hep-th]].

[5] H. Lu, J. Mei and C. N. Pope, Phys. Rev. Lett. 103, 091301 (2009) arXiv:0904.1595 [hep-th]].

[6] R. G. Cai, L. M. Cao and N. Ohta, Phys. Rev. D 80, 024003 (2009) arXiv:0904.3670 [hep-th]].

[7] R. G. Cai, Y. Liu and Y. W. Sun, JHEP 0906, 010 (2009) arXiv:0904.4104 [hep-th]].

[8] E. O. Colgain and H. Yavartanoo, JHEP 0908, 021 (2009) arXiv:0904.4357 [hep-th]].

[9] Y. S. Myung and Y. W. Kim, arXiv:0905.0179 [hep-th].

[10] A. Kehagias and K. Sfetsos, Phys. Lett. B 678, 123 (2009) arXiv:0905.0477 [hep-th]].

[11] R. G. Cai, L. M. Cao and N. Ohta, Phys. Lett. B 679, 504 (2009) arXiv:0905.0751 [hep-th]].

[12] A. Ghodsi, arXiv:0905.0836 [hep-th];

[13] Y. S. Myung, Phys. Lett. B 678, 127 (2009) [arXiv:0905.0957 [hep-th]].

[14] S. Chen and J. Jing, Phys. Lett. B 687 (2010) 124 arXiv:0905.1409 [gr-qc]]. 
[15] S. b. Chen and J. l. Jing, Phys. Rev. D 80, 024036 (2009) arXiv:0905.2055 [gr-qc]].

[16] J. Chen and Y. Wang, Int. J. Mod. Phys. A 25 (2010) 1439 [arXiv:0905.2786 [gr-qc]].

[17] M. Botta-Cantcheff, N. Grandi and M. Sturla, arXiv:0906.0582 [hep-th].

[18] M. I. Park, JHEP 0909, 123 (2009) arXiv:0905.4480 [hep-th]].

[19] A. Ghodsi and E. Hatefi, Phys. Rev. D 81 (2010) 044016 [arXiv:0906.1237 [hep-th]].

[20] A. Castillo and A. Larranaga, arXiv:0906.4380 [gr-qc].

[21] J. J. Peng and S. Q. Wu, Eur. Phys. J. C 66 (2010) 325 arXiv:0906.5121 [hep-th]].

[22] D. Y. Chen, H. Yang and X. T. Zu, Phys. Lett. B 681 (2009) 463 arXiv:0910.4821 $[\mathrm{gr}-\mathrm{qc}]]$.

[23] D. Capasso and A. P. Polychronakos, Phys. Rev. D 81 (2010) 084009 arXiv:0911.1535 [hep-th]].

[24] B. R. Majhi, Phys. Lett. B 686 (2010) 49 arXiv:0911.3239 [hep-th]].

[25] R. G. Cai and A. Wang, Phys. Lett. B 686 (2010) 166 [arXiv:1001.0155 [hep-th]].

[26] F. S. N. Lobo, T. Harko and Z. Kovacs, arXiv:1001.3517 [gr-qc].

[27] S. W. Wei, Y. X. Liu, Y. Q. Wang and H. Guo, arXiv:1002.1550 [hep-th].

[28] Y. S. Myung, arXiv:1002.4448 [hep-th].

[29] G. Koutsoumbas, E. Papantonopoulos, P. Pasipoularides and M. Tsoukalas, arXiv:1004.2289 [hep-th].

[30] Y. S. Myung, Phys. Lett. B 685 (2010) 318 arXiv:0912.3305 [hep-th]].

[31] M. Wang, J. Jing, C. Ding and S. Chen, Phys. Rev. D 81 (2010) 083006 arXiv:0912.4832 [gr-qc]].

[32] J. Greenwald, A. Papazoglou and A. Wang, arXiv:0912.0011 [hep-th].

[33] E. Kiritsis and G. Kofinas, JHEP 1001 (2010) 122 arXiv:0910.5487 [hep-th]].

[34] R. L. Arnowitt, S. Deser and C. W. Misner, The dynamics of general relativity, "Gravitation: an introduction to current research", Louis Witten ed. (Wilew 1962), chapter 7, pp 227-265, arXiv:gr-qc/0405109. 
[35] Y. S. Myung, Phys. Lett. B 684, 158 (2010) [arXiv:0908.4132 [hep-th]].

[36] A. Sen, JHEP 0509, 038 (2005) arXiv:hep-th/0506177.

[37] T. P. Sotiriou, M. Visser and S. Weinfurtner, JHEP 0910 (2009) 033 arXiv:0905.2798 [hep-th]].

[38] D. Blas, O. Pujolas and S. Sibiryakov, JHEP 0910 (2009) 029 arXiv:0906.3046 [hepth]].

[39] K. Koyama and F. Arroja, JHEP 1003 (2010) 061 [arXiv:0910.1998 [hep-th]].

[40] D. Blas, O. Pujolas and S. Sibiryakov, arXiv:0909.3525 [hep-th].

[41] A. Papazoglou and T. P. Sotiriou, Phys. Lett. B 685 (2010) 197 arXiv:0911.1299 [hep-th]].

[42] D. Blas, O. Pujolas and S. Sibiryakov, arXiv:0912.0550 [hep-th].

[43] I. Kimpton and A. Padilla, arXiv:1003.5666 [hep-th].

[44] M. Li and Y. Pang, JHEP 0908 (2009) 015 [arXiv:0905.2751 [hep-th]].

[45] M. Henneaux, A. Kleinschmidt and G. L. Gomez, Phys. Rev. D 81 (2010) 064002 arXiv:0912.0399 [hep-th]].

[46] J. Bellorin and A. Restuccia, arXiv:1004.0055 [hep-th]. 